\title{
HAMILTONIAN DYNAMICAL SYSTEMS WITHOUT PERIODIC ORBITS
}

\author{
VIKTOR L. GINZBURG
}

\begin{abstract}
The present paper is a review of counterexamples to the "Hamiltonian Seifert conjecture" or, more generally, of examples of Hamiltonian systems having no periodic orbits on a compact energy level. We begin with the discussion of the "classical" and volumepreserving Seifert conjectures. Then the constructions of counterexamples to the Hamiltonian Seifert conjecture in dimensions greater than or equal to six is outlined, mainly following the method introduced by the author of the paper. The Hamiltonian version of Seifert's theorem is also stated. A list of all known at this moment examples and constructions of Hamiltonian flows without periodic flows concludes the review.
\end{abstract}

\section{INTRODUCTION}

The existence problem for periodic orbits of vector fields or diffeomorphisms occupies one of the central places in the theory of dynamical systems and adjacent areas such as mechanics and symplectic geometry. This question is often well motivated for a particular system, but in many cases the answer gives very little information about the dynamics of the system in general. Perhaps, the methods developed to solve the existence problem are as important as the answers obtained.

The search for dynamical systems without periodic orbits has been inspired by a few questions. One of them is to determine the limits of the existence theorems. For instance, Seifert's theorem on periodic orbits of vector fields on $S^{3}$ led to the famous Seifert conjecture recently solved by K. Kuperberg, $\mathrm{KuK}$. However, in addition to this, the systems found as a result of this search sometimes exhibit a new type of dynamics and extend our understanding of the qualitative behavior that can occur for a given class of flows.

In this review we focus on examples of Hamiltonian systems without periodic orbits on a compact energy level in the context of the Seifert conjecture. The paper is organized as follows.

Date: October, 1998.

The work is partially supported by the NSF and by the faculty research funds of the University of California, Santa Cruz. 
In Section 2 we recall Seifert's theorem and the Seifert conjecture. We discuss the history of counterexamples to the Seifert conjecture from Wilson's theorem (with its proof) to the ultimate solution due to K. Kuperberg. We also briefly touch upon related results on volume-preserving flows.

Section 3 is devoted to Hamiltonian vector fields. We outline the constructions of counterexamples to the Hamiltonian Seifert conjecture in dimensions greater than or equal to six, mainly following the method introduced by the author of the present review. We also state the Hamiltonian version of Seifert's theorem.

Finally, a list of all known at this moment constructions of Hamiltonian flows without periodic flows is presented in Section 4 .

Acknowledgments. The author is deeply grateful to Ana Cannas da Silva, Carlos Gutierrez, Ely Kerman, Richard Montgomery, Marina Ratner, Andrey Reznikov, and Claude Viterbo for their advice and useful discussions. He would also like to thank the Université Paris-Sud for its hospitality during the period when the work on this manuscript was started.

\section{The Seifert Conjecture}

2.1. Seifert's Theorem. The history of examples of dynamical systems without periodic orbits as we understand it today begins with a result of Seifert that a $C^{1}$-smooth vector field on $S^{3}$ which is $C^{0}$-close to the Hopf field has at least one periodic orbit, Sei]. Later this theorem was generalized by Fuller, [Fu], as follows.

Theorem 2.1. Let $E \rightarrow B$ be a principal circle bundle over a compact manifold $B$ with $\chi(B) \neq 0$. Let also $X$ be a $C^{1}$-smooth vector field $C^{0}$-close to the field $X_{0}$ generating the $S^{1}$-action on $E$. Then $X$ has at least one periodic orbit.

Today we know two approaches to proving existence theorems for periodic orbits like Theorem 2.1 neither of which has been trivialized and made into a part of mathematical pop culture. The first approach, not counting the original Seifert proof, relies on the notion of the Fuller index, [Fu], an analogue of the Euler characteristic for periodic orbits. The second one, analytical, is due to Moser [Mo2]. Moser's method uses a version of an infinite-dimensional inverse function theorem or, more precisely, its proof. In fact, as has been recently noticed by Kerman, [Ke], under the additional assumption that $X$ is $C^{1}$-close to $X_{0}$ (but not only $C^{0}$-close), Moser's argument can be significantly simplified by applying the inverse function theorem in Banach spaces. This stronger closeness hypothesis holds in virtually all applications of the theorem known to the author. Note also that it is typical for results like Theorem 2.1 that requiring $X-X_{0}$ to be small with respect to a higher order norm often allows one to considerably simplify the proof.

A representative corollary of Theorem 2.1 (for which the $C^{1}$-closeness assumption is sufficient) is as follows: Let $f: \mathbb{R}^{2 n} \rightarrow \mathbb{R}$ be a smooth function 
having a non-degenerate minimum at the origin (say $f(0)=0$ ). Assume that all eigenvalues of $d^{2} f(0)$ are equal. Then for every sufficiently small $\epsilon>0$ the level $\{f=\epsilon\}$ carries a periodic orbit of the Hamiltonian flow of $f$. In effect, the assumption that all eigenvalues of $d^{2} f(0)$ are equal is purely technical and immaterial. Moreover, there are at least $n$ periodic orbits of the Hamiltonian flow on $\{f=\epsilon\}$. (See [We1], [Mo2], Bot].) For example, when all eigenvalues are distinct the existence of $n$ periodic orbits readily follows from the inverse function theorem.

Remark 2.2. It is interesting to point out that Seifert's theorem on $(4 n+1)$ dimensional spheres (i. e., Theorem 2.1 for the Hopf fibration $S^{4 n+1} \rightarrow$ $\mathbb{C P}^{2 n}$ ) can be proved by the standard algebraic topological methods. Namely, let $D_{x} \subset S^{4 n+1}$ with $x \in S^{4 n+1}$ be a small embedded $4 n$-dimensional disc transversal to the fibers and centered at $x$. We can choose $D_{x}$ to depend smoothly on $x$. Let $P(x)$ be the first intersection with $D_{x}$ of the integral curve of $X$ through $x$. Clearly $P(x)=x$ if and only if the integral curve closes up after one revolution along the fiber. There exists a unique vector $v_{x}$ tangent to $D_{x}$ at $x$ such that $P(x)$ lies on the geodesic in $D_{x}$ beginning at $x$ in the direction $v_{x}$ and the distance from $x$ to $P(x)$ is $\left\|v_{x}\right\|$. Hence, $v_{x}=0$ if and only if $P(x)=x$. Thus it suffices to prove that the vector field $v$ vanishes at least at one point on $S^{4 n+1}$. Note also that $v$ is normal to $X_{0}$ with respect to a suitably chosen metric and so $v$ and $X_{0}$ would be linearly independent if $v$ did not vanish.

The sphere $S^{4 n+1}$ does not admit two linearly independent vector fields. (This is a very particular case of Adams' theorem which can be proved by using the Steenrod squares, see $[\mathrm{SW}]$.) Therefore, on $S^{4 n+1}$ the field $v$ vanishes somewhere and $X$ has a periodic orbit.

2.2. The Seifert Conjecture. In the same paper, [Sei], where he proved his theorem discussed above, Seifert asked the question whether every vector field on $S^{3}$ has a periodic orbit or not. The hypothetically affirmative answer to this question has become known as the Seifert conjecture. The threedimensional sphere plays, of course, a pure historical role in the conjecture and a similar question can be asked for other manifolds and also for more restricted classes of vector fields (e. g., real-analytic, divergence-free, or Hamiltonian).

The first counterexample to the generalized Seifert conjecture is due to Wilson, Wi], who showed that the smooth Seifert conjecture fails in dimensions greater than three by proving the following

Theorem 2.3. Let $M$ be a compact connected manifold with $\chi(M)=0$ and $m=\operatorname{dim} M \geq 4$. Then there exists a smooth vector field on $M$ without periodic orbits and singular points.

Proof. Let $v_{0}$ be a vector field on $M$ without zeros. The idea is to modify $v_{0}$ so as to eliminate periodic orbits. First assume that $v_{0}$ has a finite number of periodic orbits. (Note that this is not a generic property.) Then 
each of periodic orbits can be eliminated by the following procedure which is common to many constructions of vector fields without closed integral curves.

A plug is a manifold $P=B \times I$, where $I=[-1,1]$ and $B$ is a compact manifold with boundary of dimension $m-1$, and a non-vanishing vector field $w$ on $P$ with the following properties円:

1. The boundary condition: $w=\partial / \partial t$ near $\partial P$, where $t$ is the coordinate on $I$. Thus a trajectory of $w$ can only leave the plug through $B \times\{1\}$.

2. Existence of trapped trajectories: There is a trajectory of $w$ beginning on $B \times\{-1\}$ that never exits the plug. Such a trajectory is said to be trapped in $P$.

3. Aperiodicity: $w$ has no periodic orbits in $P$. In other words, the "flow" of $w$ is aperiodic.

4. Matched ends or the entrance-exit condition: Let points $(x,-1)$, the "entrance", and $(y, 1)$, the "exit", be on the same integral curve of $w$. Then $x=y$. Hence, every trajectory of $w$ which enters and exists $P$, has its exit point right above the entrance point with $I$ being the vertical direction.

5. The embedding condition: There exists an embedding $i: P \hookrightarrow \mathbb{R}^{m}$ such that $i_{*}(w)=\partial / \partial x_{m}$ near $\partial P$. In other words, near the boundary of $P$, the embedding $i$ preserves the vertical direction.

Assuming that the plug has been constructed, we can use it to modify $v_{0}$. Namely, consider a small flow box near a point on a periodic orbit of $v_{0}$. We choose the coordinates on the box so that $v_{0}=\partial / \partial x_{m}$. Using $i$ we embed $P$ into the box so that the trapped trajectory matches the periodic orbit of $v_{0}$. Let $v$ be the vector field obtained by replacing $v_{0}$ by $w$ inside of $i(P)$. By the first property of the plug (the boundary condition), $v$ is smooth. By the third condition (aperiodicity) and the fourth (matched ends), $v$ has one periodic orbit less than $v_{0}$. By applying this procedure to every periodic orbit of $v_{0}$, we eliminate all of them.

Let us now turn to the construction of a plug. First observe that when a plug $P^{\prime}$ satisfying all of the above conditions but the entrance-exit condition is constructed, it is easy to find a genuine plug $P$. Namely, $P$ is the union of two copies of $P^{\prime}$ with the second copy put upside-down on the top of the first copy. Hence, from now on we may forget about the entrance-exit condition. This trick (with minor modifications) is common to all constructions of plugs.

The plug from [Wi], the so-called Wilson's plug, has the unit ball $D^{m-1} \subset$ $\mathbb{R}^{m-1}$ as $B$ so that the plug is automatically embedded into $\mathbb{R}^{m}$ and $t=x_{m}$. To define $w$, fix an embedding $\mathbb{T}^{2} \hookrightarrow D^{m-1} \times 0$ with a trivial normal bundle. (Hence the requirement that $m \geq 4$.) Then a tubular neighborhood $U$ of

\footnotetext{
${ }^{1}$ There are many definitions of plugs depending on the context in which they are used. See, e.g., [KuK], [KuG], KuGK], and [Gi2]. However, the differences between them seem to be more of technical than conceptual nature.
} 
$\mathbb{T}^{2}$ in $P$ is diffeomorphic to $\mathbb{T}^{2} \times D^{m-2}(\epsilon)$, where $D^{m-2}(\epsilon)$ is the $(m-2)$ dimensional ball of a small radius $\epsilon>0$. Let $w_{1}$ be an irrational vector field on $\mathbb{T}^{2}$ and $f$ a bump function on $D^{m-2}(\epsilon)$ equal to 1 at the center of the ball and vanishing near $\partial D^{m-2}(\epsilon)$. Since $\mathbb{T}^{2}$ is embedded into $D^{m-1} \times 0$, we may assume that each $y \times D^{m-2}(\epsilon), y \in \mathbb{T}^{2}$, is parallel to the vertical direction, i. e., $\partial / \partial x_{m}$ is tangent to the fibers $y \times D^{m-2}(\epsilon)$ of the tubular neighborhood $U$. Now we set

$$
w=f w_{1}+(1-f) \frac{\partial}{\partial x_{m}}
$$

on $U$ and $w=\partial / \partial x_{m}$ on $P \backslash U$. It is easy to check that $(P, w)$ satisfies the plug conditions (except the entrance-exit condition). In particular, all trapped trajectories are asymptotic to $\mathbb{T}^{2}$. This completes the construction of the plug. In what follows we will refer to $\mathbb{T}^{2}$ with an irrational flow embedded in the plug as to the core of the plug.

When the periodic orbits of the flow of $v_{0}$ are not isolated, the plug $P$ needs to be slightly modified. Namely, one can construct $P$ so that the beginnings in $B \times\{-1\}$ of trapped trajectories form a set with non-empty interior. Then a finite number of plugs are inserted into $M$ so as to interrupt every trajectory of $v_{0}$ (regardless of whether it is closed or not). As before no new periodic orbit is created but all original ones are eliminated.

Remark 2.4. In the plug used in the case where there are infinitely many periodic orbits, we clearly have $\operatorname{div} w \neq 0$. This amounts to the fact that in the volume-preserving version of Wilson's argument, one has to start with a vector field having only a finite number of periodic orbits.

The next step after Wilson's result was the construction, due to Schweitzer [Sc], of a $C^{1}$-smooth non-vanishing vector field on $S^{3}$ (or on any compact three-dimensional manifold) without periodic orbits. The proof again is by inserting plugs. Schweitzer's plug uses as the core the closure of a trajectory of the Denjoy flow on $\mathbb{T}^{2}$ instead of an irrational flow. (Hence, only $C^{1}$ smoothness.) Since the trajectory is neither dense nor closed, one can take $\mathbb{T}^{2}$ with a small open disc deleted as $B$ in order to have the embedding condition satisfied. The vector field on $P$ is then given by (1) where $w_{1}$ is the Schweitzer field on $B$ and $f$ is an appropriately chosen cut-off function. Schweitzer's construction was later improved by Harrison, Ha, to obtain a $C^{2+\epsilon}$-smooth vector field.

Finally, a major breakthrough came when K. Kuperberg, KuK, constructed a $C^{\infty}$-smooth (and even real-analytic) three-dimensional plug rendering thus a non-singular real-analytic flow without periodic orbits on every compact three-manifold. (See also [Gh] and [KuGK].) Kuperberg's plug is entirely different from those described above. One begins with a "plug" $P^{\prime}$ satisfying all the conditions of the plug but aperiodicity - there are exactly two periodic trajectories inside of $P^{\prime}$. Then one builds $P$ by inserting parts 
of $P^{\prime}$ into $P^{\prime}$ again (self-insertion) so that the vector field on the inserted parts matches the original vector field. The self-insertion is perform so as to guarantee that the resulting flow on $P$ is free of periodic orbits.

Remark 2.5. The common feature of the constructions described above (with the exception of Kuperberg's plug) is that a vector field without periodic orbits is used as the core of the plug in order to trap a trajectory. For example, the core flow is an irrational flow on the torus in Wilson's plug or the Denjoy flow in Schweitzer's plug. Hence, one vector field without periodic orbits gives rise to a multitude of such vector fields with different phase spaces but in higher dimensions (proliferation of aperiodic flows). This is also typical for methods of finding counterexamples to the Seifert conjecture in other categories.

2.3. Volume-Preserving Flows. The Seifert conjecture for this class of flows seems to be rather similar to the Seifert conjecture in the smooth category. To be more precise, as was pointed out by A. Katok, [Ka], a divergence-free smooth non-vanishing vector field $v_{0}$ on a compact manifold of dimension $m \geq 4$ can be changed into one without periodic orbits, provided $v_{0}$ has only a finite number of periodic orbits. In fact, the field $w$ given by (1) on Wilson's plug (for isolated periodic orbits) can be chosen divergencefree. This yields a smooth volume-preserving flow on $S^{2 n+1}, 2 n+1 \geq 5$, without periodic orbits.

Much less is known in dimension three. Let us state two important results due to G. Kuperberg, [KuG].

The first one is that every compact three-manifold $M$ possesses a volumepreserving $C^{\infty}$-smooth flow with a finite number of periodic orbits and no fixed points, $\mathrm{KuG}$. This follows from the fact that $M$ can be obtained from $\mathbb{T}^{3}$ by a series of Dehn surgeries (provided that $M$ is orientable). Let us equip $\mathbb{T}^{3}$ with an irrational flow. A Dehn surgery can be interpreted as the insertion of a version of a smooth volume-preserving plug $P$ into $\mathbb{T}^{3}$. These plugs differ from those described in Section 2.2 in some essential ways. The plugs $P$ are not aperiodic - each $P$ carries exactly two periodic orbits. Moreover, the flow on $P$ is not standard on the boundary to account for the Dehn-twist in the surgery. Note that this method allows one to construct a flow with exactly two periodic orbits.

The second result is that every compact three-manifold possesses a nonvanishing divergence-free $C^{1}$ vector field without periodic orbits, [KuG]. In particular, when applied to $S^{3}$, this theorem gives a volume-preserving $C^{1}$ counterexample to the Seifert conjecture. The proof of the theorem uses the previously mentioned result and a volume-preserving version of Schweitzer's plug to eliminate periodic orbits. To construct such a plug, G. Kuperberg applies, in a non-trivial way, stretching in the vertical direction to compensate for the area change resulting from the Denjoy flow. (See $\mathrm{KuG}$ for more details.) 
Remark 2.6. There is a broad class of volume-preserving flows without periodic orbits on homogeneous spaces of Lie groups. These flows arise as actions of unipotent subgroups. For example, the horocycle flow (Section 3.3) and its Hamiltonian analogues from Example 4.2 are among such flows. Deep results on the closures of orbits for these flows and on their invariant measures are obtained by Ratner. (See Rat and references therein).

\section{Hamiltonian Vector Fields without Periodic Orbits}

The question of (non-)existence of periodic orbits for Hamiltonian dynamical systems differs from that for general smooth dynamical systems in at least one important way - Hamiltonian systems tend to have a lot of periodic orbits. For example, it is reasonable to expect that a given Hamiltonian system with a proper smooth Hamiltonian has a closed orbit on every regular energy level. Taken literally, this statement is not correct in general. (For example, Zehnder, $[\mathbb{Z} \mathbb{E}]$, found a Hamiltonian flow on $\mathbb{T}^{2 n}, 2 n \geq 4$, with an irrational symplectic structure such that there are no periodic orbits for a whole interval of energy values; see Example 4.1 below.) However, periodic orbits are known to exist for almost all energy values for a broad class of symplectic manifolds. For instance, as shown by Hofer, Zehnder, and Struwe, [HZ1, St], almost all levels of a smooth proper function on $\mathbb{R}^{2 n}$ carry at least one periodic orbit. A similar theorem holds for cotangent bundles, $[\mathrm{HV}]$. (The reader interested in a detailed discussion and further references should consult HZ2].)

Furthermore, according to the $C^{1}$-closing lemma of Pugh and Robinson, [PR], a periodic orbit can be created from a recurrent trajectory by a $C^{1}$ small smooth perturbation of the original dynamical system. (As Carlos Gutierrez pointed out to the author, the perturbation can in fact be made $C^{\infty}$-smooth, Gu2.) As a consequence of the closing lemma, a $C^{1}$-generic system has the union of its periodic trajectories dense in the set of its recurrent points. Both of these results hold for Hamiltonian systems, [PR].

The situation becomes more subtle when the $C^{1}$-topology is replaced by the $C^{k}$-topology with $k>1$. The $C^{2}$-closing lemma has not been yet proved or disproved. (See, e.g., [Gu1], [Ca], and [AZ for partial results, examples, and references.) In the Hamiltonian setting, the systems whose periodic trajectories are dense may no longer be generic if $k$ is roughly speaking greater than the dimension of the manifold. For example, according M. Herman, [Her1, Her2], Hamiltonian vector fields with Hamiltonians $C^{k}$-close to Zehnder's Hamiltonian on $\mathbb{T}^{2 n}$ do not have periodic orbits for an interval of energy values, provided that the symplectic form satisfies a certain Diophantine condition and $k>2 n$. These examples are, however, in some sense exceptional. In fact, the theorems on the density of energy values for periodic orbits can be interpreted that the Hamiltonian $C^{k}$-closing lemma holds in a very strong form for many symplectic manifolds. It is still not 
known if the $C^{k}$-closing lemma with $k \geq 2$ fails in general for Hamiltonian flows when the symplectic form is exact near the energy level.

The examples of Hamiltonian flows without periodic orbits on a compact energy level are scarce. In Section 4 we attempt to list all known constructions of such flows. In this section, we focus on the Hamiltonian Seifert conjecture and on one particular method to find a Hamiltonian vector fields without periodic orbits.

Remark 3.1. There are a number of results concerning existence of periodic orbits on a fixed energy level. Recall that according to Weinstein's conjecture there is a periodic orbit on an energy level of contact type, We2]. This conjecture was proved for $\mathbb{R}^{2 n}$ by Viterbo in Vi1 and then for many other symplectic manifolds (e. g., for cotangent bundles in [HV]). The reader interested in details and up-to-date references should consult [HZ2] and [Vi2].

3.1. The Seifert Conjecture for Hamiltonian Vector Fields. The Seifert conjecture can be extended to Hamiltonian flows in a number of ways. For example, one may ask whether there is a proper smooth function on a given symplectic manifold (e.g., $\mathbb{R}^{2 n}$ ) having a regular level without periodic orbits. Equivalently, the question is whether in a given symplectic manifold there exists a regular compact hypersurface without closed characteristics. One can even ask whether there is a function with a sufficiently big set of energy levels without periodic orbits.

Recall that a characteristic of a two-form $\eta$ of rank $(2 n-2)$ on a $(2 n-1)$ dimensional manifold is an integral curve of the field of directions formed by the null-spaces ker $\eta$.

Let $i: M \hookrightarrow W$ be an embedded smooth compact hypersurface without boundary in a $2 n$-dimensional symplectic manifold $(W, \sigma)$.

Theorem 3.2. Assume that $2 n \geq 6$ and that $i^{*} \sigma$ has only a finite number of closed characteristics. Then there exists a $C^{\infty}$-smooth embedding $i^{\prime}: M \hookrightarrow W$, which is $C^{0}$-close and isotopic to $i$, such that $i^{\prime *} \sigma$ has no closed characteristics.

An irrational ellipsoid $M$ in the standard symplectic vector space $\mathbb{R}^{2 n}=$ $W$ corresponds to a collection of $n$ uncoupled harmonic oscillators whose frequencies are linearly independent over $\mathbb{Q}$, i. e., $M$ is the unit level of a quadratic Hamiltonian with rationally independent frequencies. Thus $M$ carries exactly $n$ periodic orbits. Applying Theorem 3.2, we obtain the following

Corollary 3.3. For $2 n \geq 6$, there exists a $C^{\infty}$-embedding $S^{2 n-1} \hookrightarrow \mathbb{R}^{2 n}$ such that the restriction of the standard symplectic form to $S^{2 n-1}$ has no closed characteristics.

Corollary 3.4. For $2 n \geq 6$, there exists a $C^{\infty}$-function $h: \mathbb{R}^{2 n} \rightarrow \mathbb{R}, C^{0}$-close and isotopic (with a compact support) to a positive definite quadratic form, 
such that the Hamiltonian flow of $h$ has no closed trajectories on the level set $\{h=1\}$.

Theorem 3.2 can be applied to any compact hypersurface with a finite number of closed characteristics in $\mathbb{R}^{2 n}$ instead of an irrational ellipsoid as in the corollaries. Note, however, that the only known examples of such hypersurfaces in $\mathbb{R}^{2 n}$ that are not diffeomorphic to $S^{2 n-1}$ are non-simply connected hypersurfaces constructed Laudenbach, La.

Remark 3.5. These results, proved in [Gi2] and, independently, by M. Herman [Her3], first required the ambient space to be at least eight-dimensional, i. e., $2 n \geq 8$, in the $C^{\infty}$-case. A $C^{2+\epsilon}$-hypersurface in $\mathbb{R}^{6}$ was found by $\mathrm{M}$. Herman Her3]. In Gi5], Theorem 3.2 and its corollaries were extended to $2 n=6$.

Remark 3.6. Almost nothing is known on how big the set of energy values $\mathcal{E}$ of the levels without periodic orbits can be. It is clear that as in Corollary 3.4, there exists a function on $\mathbb{R}^{2 n}$ for which $\mathcal{E}$ is infinite (but discrete). It seems plausible that $\mathcal{E}$ may have a limit point but it is not clear at all how to construct $h$ for which the set $\mathcal{E}$ would be dense.

An alternative way to formulate the Hamiltonian Seifert conjecture is to consider an odd-dimensional manifold with a maximally non-degenerate closed two-form (instead of working with the restriction of the symplectic form to a hypersurface). A result similar to Theorem 3.2, involving only two-forms on $M$ but no symplectic embedding, is also correct.

Let $\operatorname{dim} M=2 n-1$ and let $\eta$ be a closed maximally non-degenerate (i. e., of rank $(2 n-2))$ two-form on $M$. Recall that two such forms $\eta$ and $\eta^{\prime}$ are said to be homotopic if there exists a family $\eta_{\tau}, \tau \in[0,1]$, of closed maximally non-degenerate forms, all in the same cohomology class, connecting $\eta=\eta_{0}$ with $\eta^{\prime}=\eta_{1}$. The following theorem is proved in [Gi2] and Gi5].

Theorem 3.7. Assume that $2 n-1 \geq 5$ and that $\eta$ has a finite number of closed characteristics. Then there exists closed a maximally non-degenerate 2-form $\eta^{\prime}$ on $M$, homotopic to $\eta$, without closed characteristics.

Remark 3.8. In fact, Theorem 3.7 is a corollary of Theorem 3.2. To derive Theorem 3.7 from Theorem 3.2, consider a pair $\left(M^{2 n-1}, \eta\right)$, where $\eta$ closed maximally non-degenerate. Then, as Ana Cannas da Silva pointed out to the author, there exists a symplectic manifold $\left(W^{2 n}, \sigma\right)$ and a proper embedding $M \hookrightarrow W$ such that $\left.\sigma\right|_{M}=\eta$.

Indeed, let $W=M \times(-1,1)$ and let $t$ be the coordinate on $(-1,1)$. To construct $\sigma$, fix a 1-form $\alpha$ on $M$ such that ker $\alpha$ is everywhere transversal to the characteristics of $\eta$. Then $\sigma=\eta+d(\epsilon t \alpha)$ is symplectic on $W$, provided that $\epsilon>0$ is small enough.

Technically, however, it is more convenient to prove Theorem 3.7 first and then to modify its proof to obtain Theorem 3.2 . 
Remark 3.9. Theorem 3.7 extends to the real analytic case: one can make the form $\eta^{\prime}$ real analytic, provided that $Q$ and $\eta$ are real analytic. The argument is the same as that used in the construction of a real analytic version of Wilson's, Wi], or Kuperberg's, [KuK, flow. (See [Gh and KuGK.)

3.2. The Hamiltonian Seifert Theorem. Before we turn to the outline of the proofs of Theorems 3.2 and 3.7, let us state a result which can be viewed as a Hamiltonian version of Theorem 2.1.

Recall that a periodic orbit of a vector field or an integral curve of a one-dimensional foliation is called non-degenerate if the linearization of its Poincaré return map does not have unit as an eigenvalue. Let $(B, \omega)$ be a compact symplectic manifold and let $\pi: M \rightarrow B$ be a principle $S^{1}$-bundle. For a one-form $\lambda$ such that $d \lambda$ is $C^{0}$ sufficiently small, the closed two-form $\eta=\pi^{*} \omega+d \lambda$ on $M$ is maximally non-degenerate (and homotopic to $\pi^{*} \omega$ ).

Theorem 3.10 ([Gi4]). If $d \lambda$ is $C^{0}$-small enough, the number of closed characteristics of $\eta$ is strictly greater than the cup-length of $B$. If, in addition, all closed characteristics are non-degenerate, their number number is greater than or equal to the sum of Betti numbers of $B$.

Theorem 3.10 generalizes some of the results (for equal eigenvalues) of We1, Mo2, Bot]. A simple geometrical proof for the case where $B$ is a surface and $\lambda$ is $C^{2}$-small can be found in [Gi1]. Theorem 3.10 is closely related to the Arnold conjecture and to the problem of existence of periodic orbits of a charge in a magnetic field; Gi3. Note also that if $d \lambda$ is not $C^{0}$-small, but $\eta$ is still non-degenerate and homotopic to $\pi^{*} \omega$, the form $\eta$ may have no closed characteristics at all. The example is the horocycle flow described in the next section. (See Gi3] and Gi4 for more details.)

\subsection{Proofs of Theorems 3.2 and 3.7 and Symplectic Embeddings.} There are two essentially different methods to prove these theorems. Both approaches follow the same general line as Wilson's argument (i. e., the proof of Theorem 2.3), modified to make it work in the symplectic category, and vary only in the dynamics of the plug.

The plug introduced by M. Herman, Her3, is the symplectization of Wilson's plug when $2 n \geq 8$ or of the Schweitzer-Harisson plug when $2 n=6$ (hence $C^{2+\epsilon}$-smoothness constraint) modified at infinity. In other words, these plugs are obtained by taking the induced flows on the cotangent bundles to the non-Hamiltonian plugs and altering them away from a neighborhood of the zero section while keeping all the properties of the plug.

The plugs from Gi2, Gi5 are build similarly to Wilson's plug but the entire construction is Hamiltonian. Let us give some more details on this method focusing specifically the proof of Theorem 3.7. (The passage to Theorem 3.2 is simply by showing that the same argument can be carried out for hypersurfaces. See [Gi2].) 
In the construction of the plug $P$ below we identify a neighborhood in $M$ in which $P$ is embedded with a small ball in $\mathbb{R}^{2 n-1}$ equipped with the two-form $\sigma$ induced by the canonical inclusion $\mathbb{R}^{2 n-1} \subset \mathbb{R}^{2 n}$.

The key element which makes Wilson's construction work in the Hamiltonian category is that the core of the plug (i. e., the flow replacing the irrational flow on the torus) is also chosen to be Hamiltonian. In other words, to have the aperiodicity condition satisfied, we need to find a Hamiltonian flow on a symplectic manifold having no periodic orbits on some compact energy level. Furthermore, to have the embedding condition satisfied, this energy level with the induced two-form has to be embeddable in $\mathbb{R}^{2 n-1}$ to be made into a part of the plug. (In particular, the symplectic form must be exact, on a neighborhood of the energy level.) The Hamiltonian flow used [Gi2, Gi5] is the horocycle flow which we will now describe.

Let $\Sigma$ be a closed surface with a hyperbolic metric, i. e., a metric with constant negative curvature $K=-1$. Denote by $\lambda$ the canonical Liouville one-form " $p d q$ " on $T^{*} \Sigma$ and by $\Omega$ the pull-back of the area form on $\Sigma$ to $T^{*} \Sigma$. The form $\omega=d \lambda+\Omega$ is symplectic on $T^{*} \Sigma$ and exact on the complement of the zero section. The Hamiltonian flow of the standard metric Hamiltonian on $T^{*} \Sigma$ with respect to the twisted symplectic form $\omega$ is known to have no periodic orbits on the unit energy level $S T^{*} \Sigma$. Indeed, the restriction $\varphi^{t}$ of this flow to $S T^{*} \Sigma$ is the standard horocycle flow. The fact that $\varphi^{t}$ has no periodic orbits is a consequence of the classical result of Hedlund, [Hed], that the horocycle flow is minimal, i. e., all orbits are dense. We will return to the horocycle flow in Section 4 .

To construct the plug, we then need to prove that there is a "symplectic" embedding of a neighborhood $U$ of $S T^{*} \Sigma$ into $\mathbb{R}^{2 n-1}$, i. e., an embedding $j: U \hookrightarrow \mathbb{R}^{2 n-1}$ such that $j^{*} \sigma=\omega$. When $2 n-1 \geq 7$, this readily follows from a general symplectic embedding theorem due to Gromov, Gr1, Gr2.

When $2 n-1=5$ an additional argument is required. Consider the forms $\omega_{t}=d \lambda+t \Omega$ on $U$. These forms are symplectic for all $t$. Note that there exists a "symplectic" embedding $j_{0}:\left(U, \omega_{0}\right) \hookrightarrow\left(\mathbb{R}^{5}, \sigma\right)$. This is an easy consequence of the fact that there exists a Lagrangian immersion $\Sigma \rightarrow \mathbb{R}^{4}$, Gr1, Gr2]. Note also that $U$ can be replaced by a closed neighborhood of $S T^{*} \Sigma$. Then the existence of a symplectic embedding for $2 n-1=5$ is a particular case of the following result improving the dimensional constraints from [Gr1, Gr2] by one.

Let $U$ and $V$ be manifolds of equal dimensions. The manifold $U$ is assumed to be compact, perhaps with boundary, while $V$ may be open but must be a manifold without boundary. Let $\sigma$ be a symplectic form on $V$. Abusing notation, also denote by $\sigma$ the pull-back of $\sigma$ to $V \times \mathbb{R}$ under the natural projection $V \times \mathbb{R} \rightarrow V$. 
Theorem 3.11 (Gi5). Let $\omega_{t}, t \in[0,1]$, be a family of symplectic forms on $U$ in a fixed cohomology class: $\left[\omega_{t}\right]=$ const. Assume that there is an embedding $j_{0}: U \hookrightarrow V \times \mathbb{R}$ such that $j_{0}^{*} \sigma=\omega_{0}$. Then there exists an embedding $j: U \hookrightarrow V \times \mathbb{R}$, isotopic to $j_{0}$, with $j^{*} \sigma=\omega_{1}$.

Remark 3.12. Since $\left.\sigma\right|_{V}$ is symplectic, the composition of $j$ with the projection to $V$ is necessarily an immersion. When $\partial U=\emptyset$, Theorem 3.11 follows immediately from Moser's theorem Mo1].

The rest of the proof (i. e., the construction of the form $\eta^{\prime}$ on the complement of the core) proceeds according to the same scheme as the proof of Theorem 2.3 with more or less straightforward modifications; see [Gi2 for details. This completes the outline of the proofs of Theorems 3.2 and 3.7 .

\section{The List of Hamiltonian Flows Without Periodic Orbits.}

In this section we list all known to the author examples of smooth Hamiltonian systems on symplectic manifolds $(V, \omega)$ having no periodic orbits on a compact regular level $M$. This list in similar to the one given in Gi5 with the exception of Example 4.2.

The list is divided into two parts according to whether $\omega$ is exact near $M$ or not. The reason for this division is that flows for which $\left[\left.\omega\right|_{M}\right] \neq 0$ can be expected to have a qualitative behavior different from those with $\left[\left.\omega\right|_{M}\right]=0$. For instance, Zehnder's flow appears to be more robust than seems to be possible for "exact" flows.

Case 1: The form $\omega$ is not exact.

Example 4.1 (Zehnder's torus, $[\bar{Z}]$ ). Let $2 n \geq 4$. Consider the torus $V=$ $\mathbb{T}^{2 n}$ with an irrational translation-invariant symplectic structure $\omega$. Choose a Hamiltonian $H$ on $V$ so that every level $\{H=c\}$ with $c \in(0.5,1.5)$ is the union of two standard embedded tori $\mathbb{T}^{2 n-1} \subset \mathbb{T}^{2 n}$. Since $\omega$ is irrational, the characteristics of $\left.\omega\right|_{\mathbb{T}^{2 n-1}}$ form an quasiperiodic flow on $\mathbb{T}^{2 n-1}$. Thus, none of the levels $\{H=c\}$ with $c \in(0.5,1.5)$ carries a periodic orbit. Note that the restriction of $\omega$ to these energy levels is never exact. As we have already pointed out, the flow in question exhibits remarkable stability properties according to a result of M. Herman, Her1, Her2].

Example 4.2 (The Hamiltonian horocycle flow in dimension $2 n$ ). Let $\mathbb{C} H^{n}$ be the complex hyperbolic space equipped with its standard Kähler metric (see, e.g., Section XI.10 of [KN]). Pick a discrete subgroup $\Gamma$ in the group $\mathrm{SU}(1, n)$ of Hermitian isometries of $\mathbb{C} H^{n}$ such that $B=\Gamma \backslash \mathbb{C} H^{n}$ is smooth and compact. (To see that $\Gamma$ exists recall that according to a theorem of Borel, [Bor], there is a discrete subgroup $\Gamma$ such that $\Gamma \backslash \mathbb{C} H^{n}$ is compact. Then by Selberg's lemma, Se]], $\Gamma$ can be chosen so that $B$ is smooth.) 
Let $H$ be the standard metric Hamiltonian and let $d \lambda$ be the standard symplectic structure on $T^{*} B$. Denote by $\Omega$ the pull-back to $T^{*} B$ of the Kähler symplectic form on $B$. The Hamiltonian flow of $H$ with respect to the twisted symplectic structure $\omega=\Omega+d \lambda$ has no periodic orbits on the level $M=\{H=1\}$. (This follows from the fact this flow is generated by the (right) action of a unipotent one-parameter subgroup of $\mathrm{SU}(1, n)$ and that $\Gamma$ contains no unipotent elements, [KM]; see also [Rag], [Rat], and references therein.)

When $n=1$ this construction gives exactly the horocycle flow on the unit (co)tangent bundle to a surface $B=\Sigma$. If $n>1$, the form $\omega$ is not exact in a neighborhood of $M$.

Note also that these Hamiltonian horocycle flows arise in the description of the motion of a charge in a magnetic field on the configuration space $B$; see Gi3, Gi4]. These are the only known "magnetic" flows without periodic orbits on an energy level.

Case 2: The form $\omega$ is exact near the energy level. In this case we should distinguish whether $\operatorname{dim} V=4$ or $\operatorname{dim} V \geq 6$.

When $\operatorname{dim} V=4$, the only known smooth example is the horocycle flow described as a Hamiltonian system in Section 3.3 and in Example 4.2 with $n=1$ and $B=\Sigma$. To slightly generalize this construction, note that a neighborhood of $S T^{*} \Sigma$ can be identified with $V=\Gamma \backslash \mathrm{SU}(1,1) \times(1-$ $\epsilon, 1+\epsilon)$, where $\Gamma=\pi_{1}(\Sigma)$, so that $H$ becomes the projection to the second component. Then, instead of $\Gamma=\pi_{1}(\Sigma)$ we can take any discrete subgroup such that the quotient $\Gamma \backslash \mathrm{SU}(1,1)$ is compact and smooth. As follows from Remark 3.8, the flow on $\Gamma \backslash \mathrm{SU}(1,1)$ generated by the action of a unipotent subgroup of $\mathrm{SU}(1,1)$ is the Hamiltonian flow of $H$ on $\{H=1\}$ with respect to some symplectic form.

Finally note that by Remark 3.8 the flows on three-manifolds constructed by G. Kuperberg and described above in Section 2.3 can also be thought of as Hamiltonian flows on some symplectic manifolds. This gives a class of smooth Hamiltonian flows on symplectic manifolds with a finite number of closed orbits on a given energy level or $C^{1}$-flows without periodic orbits. It is not known whether such a flow on $S^{3}$ can be obtained by a $C^{2}$-embedding of $S^{3}$ into $\mathbb{R}^{4}$.

When $\operatorname{dim} V \geq 6$, the only known examples are essentially those described in Section 3.1 or those obtained by iterations of their constructions. In other words, beginning with a flow with a finite number of periodic orbits one can eliminate these orbits by using the plugs from [Gi2, Gi5] or Her3]. The resulting manifold can also be used as the core of a plug in the same way as the horocycle flow. As in Remark 2.5, these new plugs can in turn be employed to construct Hamiltonian flows without periodic orbits, etc.

Since the starting point of this method is a flow with a finite number of periodic orbits, it makes sense to ask how many such flows are known in addition to those on irrational ellipsoids. For example, we have already 
mentioned non-simply connected hypersurfaces in $\mathbb{R}^{2 n}$ found by Laudenbach [La]. To produce more examples, we can apply the same method as described in the previous paragraph. Namely, if any Hamiltonian flow with a finite number of periodic orbits can be used as the core of the plug, the resulting "plug" will also have only a finite number of periodic orbits inside of it. Thus by inserting it into another flow with a finite number of periodic orbits, we can create yet one more flow with the same property. For example, by taking $S^{1} \subset \mathbb{R}^{2}$ as the core, Cieliebak, $\mathrm{Ci}$, constructed embeddings $S^{2 n-1} \subset \mathbb{R}^{2 n}$, $2 n \geq 4$, such that pairs of closed characteristics are linked and knotted in an essentially arbitrary way. (Each plug results into two "parallel" closed orbits, but there are no constraints on knotting of these pairs or their linking.)

\section{REFERENCES}

[AZ] Aranson, S., Zhuzhoma, E., On the $C^{r}$-closing lemma on surfaces, Russian Math. Surv., 43 (1988), no. 5, 209-210.

[Bor] Borel, A., Compact Clifford-Klein forms of symmetric spaces, Topology, 2 (1963), 111-122.

[Bot] Bottkol, M., Bifurcation of periodic orbits on manifolds and Hamiltonian systems. J. Diff. Eq., 37 (1980), 12-22.

[Ca] Carroll, C., Rokhlin towers and $C^{r}$ closing for flows on $T^{2}, E r g$. Th. and Dynam. Sys., 12 (1992), 683-706.

[Ci] Cieliebak, K., Symplectic boundaries: Creating and destroying closed characteristics, GAFA, 7 (1997), 269-321.

[Fu] Fuller, F. B., An index of fixed point type for periodic orbits, Amer. J. Math., 89 (1967), 133-148.

[Gi1] Ginzburg, V. L., New generalizations of Poincaré's geometric theorem, Funct. Anal. Appl., 21 (2) (1987), 100-106.

[Gi2] Ginzburg, V. L., An embedding $S^{2 n-1} \rightarrow \mathbb{R}^{2 n}, 2 n-1 \geq 7$, whose Hamiltonian flow has no periodic trajectories, IMRN, (1995), no. 2, 83-98.

[Gi3] Ginzburg, V. L., On closed trajectories of a charge in a magnetic field. An application of symplectic geometry, in Contact and Symplectic Geometry, C.B. Thomas (Editor), Publications of the Newton Institute, Cambridge University Press, Cambridge, 1996, p. 131-148.

[Gi4] Ginzburg, V. L., On the existence and non-existence of closed trajectories for some Hamiltonian flows, Math. Z., 223 (1996), 397-409.

[Gi5] Ginzburg, V. L., A smooth counterexample to the Hamiltonian Seifert conjecture in $\mathbb{R}^{6} . I M R N,(1997)$, no. 13, 641-650.

[Gr1] Gromov, M., A topological technique for the construction of solution of differential equations and inequalities, ICM. 1970, Nice, vol. 2, pp. 221-225.

[Gr2] Gromov, M., Partial differential relations, Springer Verlag, New York, 1986.

[Gu1] Gutierrez, C., A counter-example to a $C^{2}$-closing lemma, Erg. Th. and Dyn. Sys., 7 (1987), 509-530.

[Gu2] Gutierrez, C., Letter to the author, 1998.

[Gh] Ghys, E., Construction de champs de vecteurs sans orbite périodique (d'après Krystyna Kuperberg), Sém. Bourbaki, 1993-1994, no 785, Juin 1994.

[Ha] Harrison, J., A $C^{2}$ counterexample to the Seifert conjecture. Topology, 27 (1988), 249-278.

[Hed] Hedlund, G. A., Fuchsian groups and transitive horocycles, Duke Math. J., 2 (1936), 530-542. 
[Her1] Herman, M.-R., Examples de flots hamiltoniens dont aucune perturbations en topologie $C^{\infty}$ n'a d'orbites périodiques sur ouvert de surfaces d'énergies, $C$. $R$. Acad. Sci. Paris Sér. I Math., 312 (1991), 989-994.

[Her2] Herman, M.-R., Différentiabilité optimale et contre-exemples á la fermeture en topologie $C^{\infty}$ des orbites récurrentes de flots hamiltoniens. C. R. Acad. Sci. Paris Sér. I Math., 313 (1991), 49-51.

[Her3] Herman, M.-R., Fax to Eliashberg.

[HV] Hofer, H., Viterbo, C., The Weinstein conjecture for cotangent bundles and related results, Ann. Scuola Norm. Sup. Pisa Cl. Sci., (4) 15 (1988) no. 3, 411-445 (1989).

[HZ1] Hofer, H., Zehnder, E., Periodic solution on hypersurfaces and a result by C. Viterbo, Invent. Math., 90 (1987), 1-9.

[HZ2] Hofer, H., Zehnder, E., Symplectic invariants and Hamiltonian dynamics, Birkhäuser, Advanced Texts; Basel-Boston-Berlin, 1994.

[Ka] Katok, A., Private communication, 1990.

[KM] Kazhdan, D. A., Margulis, G. A., A proof of Selberg's conjecture, Mat. Sbornik (N.S.), 75 (1968), no. 2, 162-168 (in Russian).

[Ke] Kerman, E., Private communication, 1998.

[KN] Kobayashi, S., Nomizu, K., Foundations of differential geometry, Interscience Publishers, New York; vol. I, 1963; vol. II, 1969.

[KuG] Kuperberg, G., A volume-preserving counterexample to the Seifert conjecture, Comment. Math. Helv., 71 (1996), 70-97.

[KuGK] Kuperberg, G., Kuperberg, K., Generalized counterexamples to the Seifert conjecture, Ann. Math., 144 (1996), 239-268.

[KuK] Kuperberg, K., A smooth counterexample to the Seifert conjecture in dimension three, Ann. Math., 140 (1994), 723-732.

[La] Laudenbach, F., Trois constructions an topologie symplectique, Ann. Fac. Sci. Toulouse Math., 6 (1997), 697-709.

[Mo1] Moser, J., On the volume elements on a manifold, Trans. Amer. Math. Soc., 120 (1965), 286-294.

[Mo2] Moser, J., Periodic orbits near an equilibrium and a Theorem by Alan Weinstein, Comm. Pure and Appl. Math., 29 (1976), 727-747.

[PR] Pugh, C., Robinson, C., The $C^{1}$ closing lemma, including Hamiltonians, Ergod. Th. and Dynam. Sys., 3 (1983), 261-313.

[Rag] Raghunathan, M. S., Discrete subgroups of Lie groups, Springer Verlag, New York, 1972.

[Rat] Ratner, M., On Raghunathan's measure conjecture, Ann. Math. (2), 134 (1991), 545-607.

[Sc] Schweitzer, P. A., Counterexamples to the Seifert conjecture and opening closed leaves of foliations, Ann. Math., 100 (1970), 229-234.

[Sei] Seifert, H., Closed integral curves in the 3-space and 2-dimensional deformations, Proc. Amer. Math. Soc., 1 (1950), 287-302.

[Sel] Selberg, A., On discontinuous groups in higher-dimensional symmetric spaces, Contributions to function theory (Internat. Colloq. Function Theory, Bombay, 1960), 147-164, Tata Institute of Fundamental Research, Bombay, 1960.

[SW] Steenrod, N. E., Whitehead, J.H.C., Vector fields on the $n$-sphere, Proc. Nat. Acad. Sci. USA, 37 (1951), 58-63.

[St] Struwe, M., Existence of periodic solutions of Hamiltonian systems on almost every energy surfaces, Bol. Soc. Bras. Mat., 20 (1990), 49-58.

[Vi1] Viterbo, C., A proof of Weinstein's conjecture in $\mathbb{R}^{2 n}$, Ann. Inst. H. Poincaré, Anal. Non Linéaire, 4 (1987), 337-356. 
[Vi2] Viterbo, C., Functors and computations in Floer cohomology. Part I, Preprint, Université Paris-Sud, 1997.

[We1] Weinstein, A., Normal modes for non-linear Hamiltonian systems, Invent. Math., 20 (1973), 377-410.

[We2] Weinstein, A., On the hypothesis of Rabinowitz' periodic orbit theorem, J. Diff. Eq., 33 (1979), 353-358.

[Wi] Wilson, F., On the minimal sets of non-singular vector fields, Ann. Math., 84 (1966), 529-536.

[Ze] Zehnder, E., Remarks on periodic solutions on hypersurfaces, in Periodic solutions of hamiltonian systems and related topics by Rabinowitz et al., Reidel Publishing Co. (1987), 267-279.

Department of Mathematics, UC Santa Cruz, Santa Cruz, CA 95064

E-mail address: ginzburg@math.ucsc.edu 